MATHEMATICS OF COMPUTATION

Volume 72, Number 243, Pages 1239-1250

S 0025-5718(02)01469-2

Article electronically published on October 29, 2002

\title{
ON THE CONVERGENCE OF HIGH RESOLUTION METHODS WITH MULTIPLE TIME SCALES FOR HYPERBOLIC CONSERVATION LAWS
}

\author{
ROBERT KIRBY
}

\begin{abstract}
A class of finite volume methods based on standard high resolution schemes, but which allows spatially varying time steps, is described and analyzed. A maximum principle and the TVD property are verified for general advective flux, extending the previous theoretical work on local time stepping methods. Moreover, an entropy condition is verified which, with sufficient limiting, guarantees convergence to the entropy solution for convex flux.
\end{abstract}

\section{INTRODUCTION}

Hyperbolic conservation laws model a wide range of physically important phenomena in gas dynamics, shallow water hydrodynamics, and porous media applications. Throughout these problems, nonlinearities and irregular physical properties give rise to spatially varying advective velocities and discontinuous solution profiles. Upwind finite volume and finite difference methods accurately resolve the local features, but their explicit time stepping schemes have a stable time step which varies inversely with the global maximum of the advective velocity. Thus, strong local variation in the velocity can render the time discretizations inefficient. Additionally, the maximum time step also varies linearly with the characteristic mesh size, so local mesh refinement further complicates the issue.

The literature contains several approaches which seek to address this problem. Multiple grid methods, such as in [1], are widely implemented to handle the varying time scales introduced by local mesh refinement. They have the advantage of only requiring the implementation of uniform mesh calculations on each mesh plus some mechanism for communicating information between the meshes. However, a wide variety of time scales can appear outside the context of adaptive mesh codes. It is conceivable that a more general way of distributing the local time steps would have some advantage, especially in the context of unstructured meshes.

Another approach, developed more recently, introduces a space-time discretization. Examples of such an approach appear in 6, 8. These methods allow the size of the elements in the temporal direction to vary throughout space. However, they require mesh generation in one extra dimension, and enforcing stability on irregular space-time discretizations is not yet clear.

Received by the editor May 10, 2001 and, in revised form, November 30, 2001.

2000 Mathematics Subject Classification. Primary 35L65, 65M12, 65M30.

Key words and phrases. Spatially varying time steps, upwinding, conservation laws.

Supported by the ASCI/Alliances Center for Astrophysical Thermonuclear Flashes at the University of Chicago under DOE subcontract B341495. 
Single grid methods avoid some of the complications associated with the spacetime methods, while still allowing time steps to vary independently from the mesh. In addition, they inherit some of the standard methodology of finite volume and finite element methods. The work of Osher and Sanders [11] introduces a monotone finite volume method which has a main time step which could be larger than the global CFL time step, with each element still satisfying a local CFL condition. Dawson [3] formulates a two-dimensional, high resolution version of this method through slope limiters. He applies it to some simple porous media problems, observing numerical stability and accuracy comparable to that of global time stepping methods. In addition, a second order in time method is introduced in [4, and maximum principles are proven for the high resolution first and second order methods in a single space dimension. These results as well as numerical results for a recursive, multilevel implementation of these methods appear in [7]. A similar scheme has been implemented for adaptive, parallel discontinuous Galerkin methods in [5], but it fails to maintain the conservation of the method.

While devising these local time stepping schemes, it is vital to preserve the stability, accuracy, and convergence properties which motivate the use of the finite volume methods. This paper pushes forward the theory of local time stepping schemes. On one hand, it adds significant theoretical results to those established in [7] and [4]. Namely, a bound on the total variation and an entropy condition are verified for high resolution schemes under local CFL restrictions. On the other hand, this paper extends the work of [11] by generalizing that monotone local time stepping scheme to a wide class of high resolution schemes.

This paper is outlined as follows. First in Section 2 the general high resolution local time stepping method is described. Then in Section 3 a maximum principle is established under a local CFL condition. This result improves the result of [4] to a more general set of methods. In Section 4 the total variation analysis in [11] is generalized to high resolution methods. A final result in Section [5 is the proof of an entropy condition of the form

$$
\int_{\Re \times \Re+} V(w) \phi_{t}+F(w) \phi_{x} d x d t \leq-\int_{\Re} V\left(w_{0}\right) \phi(x, 0) d x .
$$

This derivation generalizes that in [9] to local time stepping methods. Additionally, the conditions in [10] for a semidiscrete MUSCL scheme for equations with convex flux to converge to the entropy solution can be naturally adapted to the local time stepping context.

\section{PROBlem AND METHOD DESCRIPTION}

Consider the scalar, one-dimensional conservation law

$$
\begin{aligned}
& u_{t}+f(u)_{x}=0, \\
& u(x, 0)=u_{0}(x),
\end{aligned}
$$

where $f$ is a Lipschitz advective flux.

Partition of the real line into intervals $I_{j}=\left\{x: x_{j-\frac{1}{2}} \leq x<x_{j+\frac{1}{2}}\right\}$. For a quantity $c$, let $c_{j}$ denote its average value on $I_{j}$. Let the operator $\Delta_{+}$denote a forward difference. That is, $\Delta_{+} c_{j} \equiv c_{j+1}-c_{j}$. Partition $[0, T)$ into intervals $\left[t^{n}, t^{n+1}\right), n=0, \ldots, N-1$ with $t^{0}=0$ and $t^{N}=T$. Let $\Delta t^{n} \equiv t^{n+1}-t^{n}$ and define $\lambda_{j}^{n} \equiv \frac{\Delta t^{n}}{\Delta x_{j}}$. 
A wide range of finite volume methods may be expressed in the forward Euler form

$$
U_{j}^{n+1}=U_{j}^{n}+C_{j}^{n} \Delta_{+} U_{j-1}^{n}+D_{j}^{n} \Delta_{+} U_{j}^{n} .
$$

Here, $C_{j}$ and $D_{j}$ are the coefficients of either a slope limited or flux limited finite volume scheme such as the MUSCL scheme [13] or any of those appearing in 12. The time step $\Delta t^{n}$ is included in the definition of the coefficients. For these methods, sufficient conditions for a maximum principle are $C_{j}^{n} \leq 0 \leq D_{j}^{n}$ and $1+C_{j}^{n}-D_{j}^{n} \geq 0$ for all $j$ and $n$. Similar conditions ensure the method is TVD [6].

For slope limiter and DG methods, $C_{j}$ and $D_{j}$ depend on a two-point numerical flux $h$ which satisfies

- $h(c, c)=f(c)$ (consistency),

- $h(\cdot, \cdot)$ is nondecreasing in the first argument and nonincreasing in the second argument (monotonicity),

- $h$ is Lipschitz continuous in both arguments.

Examples of such fluxes include the Godunov flux and the Lax-Friedrichs flux.

For local time stepping, it is necessary to further partition the time steps on certain elements as in [11. Let $\mathcal{C}^{n}$ denote the set of all indices $j$ such that a single time step is taken from $t^{n}$ to $t^{n+1}$ on $I_{j}$. On the rest of the elements, partition the time step $\left[t^{n}, t^{n+1}\right)$ into the union of substeps $\left[t^{n+\eta_{l}}, t^{n+\eta_{l+1}}\right), l=0, \ldots, M-1$. Define numbers $\eta_{l}=\sum_{k=1}^{l} \sigma_{k}$, where $\left\{\sigma_{k}\right\}_{k=1}^{M}$ is a sequence of positive numbers summing to unity. Let $\eta_{0}=0$. The sublevels in the time interval are $t^{n+\eta_{l+1}}=$ $t^{n+\eta_{l}}+\sigma_{l+1} \Delta t^{n}$. Notice that the elements on which the local steps are taken may change over time, which is essential in solving nonlinear problems. Finally, let

$$
\Lambda_{j}^{n+\eta_{l}} \equiv\left\{\begin{array}{lr}
\lambda_{j}^{n}, & j, j \pm 1 \in \mathcal{C}^{n}, \\
\sigma_{l+1} \lambda_{j}^{n}, & \text { otherwise. }
\end{array}\right.
$$

2.1. Localized finite volume method. The class of methods expressed by (2.2) may be generalized to a class of two-level methods in the spirit of [11].

$$
\begin{gathered}
U_{j}^{n+\eta_{k}}= \begin{cases}U_{j}^{n} & \text { if } j \in \mathcal{C}^{n}, \\
U_{j}^{n}+\sum_{l=0}^{k-1} \sigma_{l+1}\left[C_{j}^{n+\eta_{l}} \Delta_{+} U_{j-1}^{n+\eta_{l}}+D_{j}^{n+\eta_{l}} \Delta_{+} U_{j}^{n+\eta_{l}}\right] & \text { if } j \notin \mathcal{C}^{n} .\end{cases} \\
U_{j}^{n+1}=U_{j}^{n}+\sum_{l=0}^{M-1} \sigma_{l+1}\left[C_{j}^{n+\eta_{l}} \Delta_{+} U_{j-1}^{n+\eta_{l}}+D_{j}^{n+\eta_{l}} \Delta_{+} U_{j}^{n+\eta_{l}}\right] .
\end{gathered}
$$

Here, $C_{j}$ and $D_{j}$ are some coefficients from one of the methods discussed above, and they will satisfy local CFL restrictions rather than global ones. As in the uniform time stepping case, the condition $C_{j} \leq 0 \leq D_{j}$ must hold.

The high resolution method in 4 may be expressed in this abstract setting with $C_{j}$ and $D_{j}$ defined as

$$
\begin{aligned}
& C_{j}^{n+\eta_{l}}=-\lambda_{j}^{n} \alpha_{j, 1}^{n+\eta_{l}}\left(1+\frac{\Delta_{+} \tilde{U}_{j-1}^{n+\eta_{l}}}{\Delta_{+} U_{j-1}^{n+\eta_{l}}}\right), \\
& D_{j}^{n+\eta_{l}}=-\lambda_{j}^{n} \alpha_{j, 2}^{n+\eta_{l}}\left(1-\frac{\Delta_{+} \tilde{\tilde{U}}_{j}^{n+\eta_{l}}}{\Delta_{+} U_{j}^{n+\eta_{l}}}\right),
\end{aligned}
$$


where

$$
\alpha_{j, 1}^{n+\eta_{l}} \equiv \frac{h\left(U_{j+\frac{1}{2}, L}^{n+\eta_{l}}, U_{j+\frac{1}{2}, R}^{n+\eta_{l}}\right)-h\left(U_{j-\frac{1}{2}, L}^{n+\eta_{l}}, U_{j+\frac{1}{2}, R}^{n+\eta_{l}}\right)}{U_{j+\frac{1}{2}, L}^{n+\eta_{l}}-U_{j-\frac{1}{2}, L}^{n+\eta_{l}}}
$$

and

$$
\alpha_{j, 2}^{n+\eta_{l}} \equiv \frac{h\left(U_{j-\frac{1}{2}, L}^{n+\eta_{l}}, U_{j+\frac{1}{2}, R}^{n+\eta_{l}}\right)-h\left(U_{j-\frac{1}{2}, L}^{n+\eta_{l}}, U_{j-\frac{1}{2}, R}^{n+\eta_{l}}\right)}{U_{j+\frac{1}{2}, R}^{n+\eta_{l}}-U_{j-\frac{1}{2}, R}^{n+\eta_{l}}}
$$

are the local Lipschitz coefficients of the numerical flux $h$ and where $U_{j \pm \frac{1}{2}, L}$ etc. are computed through some slope limited reconstruction procedure.

Under one interpretation, the discontinuous Galerkin method of Cockburn and Shu [2] is simply a way of constructing slopes. To compute these slopes however, additional terms appear in the variational formuation for advancing the whole function forward in time. A local time stepping scheme must specify how these higher order moments advance in time as well as just the cell centers. For such a formulation, see [7].

2.2. Some remarks. In this section, several comments are made regarding the implementation and interpretation of this time stepping scheme. These are meant to show that the method is neither difficult to conceive nor a strange approach to handling multiple time scales in advection problems.

First, the implementation of this method centers around handling the fluxes at the interfaces between different time steps properly. All that is required is to advance the solution in the small time step $M$ times, using the solution at time $t^{n}$ in the elements with large time steps for the Riemann solution at interfaces. In slope limited methods, the slopes in the interface element with the large time step must be updated at each substep to ensure the CFL condition holds. The flux computed at the interface over a given time step must be accumulated and averaged. This average flux is used as the flux at that edge on the element with the large time step. In this way, the time integral average over a main time step $\Delta t^{n}$ is preserved at interfaces between elements with different time steps.

An existing code can be modified to incorporate this approach by flagging each element as to whether it takes large or small time steps. Routines which compute slopes, fluxes, etc. can then be rewritten to only operate on elements with a flag set to a certain value. Then, a routine which advances the solution for all elements with a given flag simply loops over the small stepping elements, advancing the solution and storing the flux at interfaces in a buffer. Before advancing the solution in the rest of the region, the flux is overwritten with the flux from this buffer. It is worth noting that this approach to implementing the method applies also to multidimensional codes. All that is required is a buffer to store fluxes at interfaces and a flag for each element. It has been successfully coded on unstructured triangular meshes for scalar problems.

It should also be noted that this approach to time stepping, in addition to the mathematical framework presented in the rest of this paper, also corresponds well to physical and numerical intuition. In explicit time stepping methods, the fastest features must be fully resolved. However, in regions with larger allowable time steps, the flow evolves relatively slowly, so approximating it in the short term with a constant seems reasonable. Further, hyperbolic equations have finite propogation 
speeds, so for small amounts of time, only a small region of the domain affects the solution at a given place. This gives freedom to resolve the flow locally at appropriate time scales.

\section{MAXIMUm PRINCIPLE}

The maximum principle argument in [11 is valid only for monotone methods, and the analysis of 4 takes a path which generlizes the argument for slope limited schemes to local time stepping. In this section, a more general maximum principle is presented that works for the general family of methods (2.4)-2.5) rather than the specific choice of $C_{j}$ and $D_{j}$ analyzed in [4. The conditions derived on $C_{j}$ and $D_{j}$ are natural localizations of the conditions on global time stepping methods discussed above.

Proposition 3.1. The general localized finite volume method (2.4)-(2.5) satisfies the maximum principle

$$
\left|U_{j}^{n}\right| \leq \sup _{j}\left|U_{j}^{0}\right|
$$

for each $j, n$ under the $C F L$ condition

$$
1+\alpha C_{j}^{n+\eta_{l}}-\beta D_{j}^{n+\eta_{l}} \geq 0,
$$

for all $0 \leq \alpha, \beta \leq \frac{\Lambda_{j}^{n+\eta_{l}}}{\lambda_{j}^{n}}$.

Proof. The proof begins by establishing the maximum principle for a given substep for $j \notin \mathcal{C}^{n}$. Using (2.4),

$$
\begin{aligned}
U_{j}^{n+1}= & U_{j}^{n+\eta_{M-1}}+\sigma_{M} C_{j}^{n+\eta_{M-1}} \Delta_{+} U_{j-1}^{n+\eta_{M-1}}+\sigma_{M} D_{j}^{n+\eta_{M-1}} \Delta_{+} U_{j}^{n+\eta_{M-1}} \\
= & \left(1+\sigma_{M} C_{j}^{n+\eta_{M-1}}-\sigma_{M} D_{j}^{n+\eta_{M-1}}\right) U_{j}^{n+\eta_{M-1}} \\
& -\sigma_{M} C_{j}^{n+\eta_{M-1}} U_{j-1}^{n+\eta_{M-1}}+\sigma_{M} D_{j}^{n+\eta_{M-1}} U_{j+1}^{n+\eta_{M-1}} .
\end{aligned}
$$

Taking the absolute value of each side and applying the CFL condition (3.2) leads to

$$
\left|U_{j}^{n+1}\right| \leq \max _{k \in[j-1, j+1]}\left|U_{k}^{n+\eta_{M-1}}\right| .
$$

This argument may be applied $M$ times to establish that for $j \notin \mathcal{C}^{n},\left|U_{j}^{n+1}\right|$ is bounded by $\max _{k \in[j-M, j+M]}\left|U_{k}^{n}\right|$.

Consider now the case of $j \in \mathcal{C}^{n}$. Using (2.4) in (2.5),

$$
\begin{aligned}
U_{j}^{n+1} & =U_{j}^{n}+\sum_{l=0}^{M-1} \sigma_{l+1}\left[C_{j}^{n+\eta_{l}} \Delta_{+} U_{j-1}^{n+\eta_{l}}+D_{j}^{n+\eta_{l}} \Delta_{+} U_{j}^{n+\eta_{l}}\right] \\
& =\sum_{l=1}^{M-1} \sigma_{l+1}\left[\left(1+C_{j}^{n+\eta_{l}}-D_{j}^{n+\eta_{l}}\right) U_{j}^{n+\eta_{l}}-C_{j}^{n+\eta_{l}} U_{j-1}^{n+\eta_{l}}+D_{j}^{n+\eta_{l}} U_{j+1}^{n+\eta_{l}}\right],
\end{aligned}
$$

so that, using (3.2), (3.4) and (2.4),

$$
\left|U_{j}^{n+1}\right| \leq \max _{k \in[j-M, j+M]}\left|U_{j}^{n}\right|,
$$

thus completing the proof. 


\section{TVD ANALYSIS}

It is well known that total variation boundedness serves as a compactness criterion giving weak convergence of the numerical solution to a weak solution of the conservation law. In this section, the analysis in [11] is extended to include high resolution methods. This appears to be the first total variation analysis of any high resolution local time stepping method.

Proposition 4.1. The method (2.4)-(2.5) is TVD under the CFL condition

$$
1+\alpha C_{j+1}^{n+\eta_{l}}-\beta D_{j}^{n+\eta_{l}} \geq 0
$$

where $0 \leq \alpha \leq \frac{\Lambda_{j+1}^{n+\eta_{l}}}{\lambda_{j+1}^{n+\eta_{l}}}$ and $0 \leq \beta \leq \frac{\Lambda_{j}^{n+\eta_{l}}}{\lambda_{j}^{n}}$.

Proof. First, the inequality

$$
\begin{aligned}
\left|U_{j+1}^{n+1}-U_{j}^{n+1}\right| \leq & \left|U_{j+1}^{n}-U_{j}^{n}\right| \\
& +\sum_{l=0}^{M-1} \sigma_{l+1} \Delta_{+}\left(C_{j}^{n+\eta_{l}}\left|\Delta_{+} U_{j-1}^{n+\eta_{l}}\right|\right) \\
& +\sum_{l=0}^{M-1} \sigma_{l+1} \Delta_{+}\left(D_{j}^{n+\eta_{l}}\left|\Delta_{+} U_{j}^{n+\eta_{l}}\right|\right)
\end{aligned}
$$

will be established for each of the cases $j, j+1 \in \mathcal{C}^{n} ; j, j+1 \notin \mathcal{C}^{n} ; j \in \mathcal{C}^{n}, j+1 \notin \mathcal{C}^{n}$; and $j \notin \mathcal{C}^{n}, j+1 \in \mathcal{C}^{n}$.

To begin, suppose that $j, j+1 \in \mathcal{C}^{n}$. Using (2.4) and grouping terms,

$$
\begin{aligned}
\left|U_{j+1}^{n+1}-U_{j}^{n+1}\right|=\mid & \mid \sum_{l=0}^{M-1} \sigma_{l+1} \\
& {\left[\left(1+C_{j+1}^{n+\eta_{l}}-D_{j}^{n+\eta_{l}}\right) \Delta_{+} U_{j}^{n+\eta_{l}}\right.} \\
& \left.\quad-C_{j}^{n+\eta_{l}} \Delta_{+} U_{j-1}^{n+\eta_{l}}+D_{j+1}^{n+\eta_{l}} \Delta_{+} U_{j+1}^{n+\eta_{l}}\right] \mid .
\end{aligned}
$$

Now, using the CFL condition (4.1) and (2.4)

$$
\begin{aligned}
\left|U_{j+1}^{n+1}-U_{j}^{n+1}\right| \leq & \sum_{l=0}^{M-1} \sigma_{l+1}\left|\Delta_{+} U_{j}^{n+1}\right| \\
& +\sum_{l=0}^{M-1} \sigma_{l+1} \Delta_{+}\left(C_{j}^{n+\eta_{l}}\left|\Delta_{+} U_{j-1}^{n+\eta_{l}}\right|\right) \\
& +\sum_{l=0}^{M-1} \sigma_{l+1} \Delta_{+}\left(D_{j}^{n+\eta_{l}}\left|\Delta_{+} U_{j}^{n+\eta_{l}}\right|\right) \\
= & \left|U_{j+1}^{n}-U_{j}^{n}\right| \\
& +\sum_{l=0}^{M-1} \sigma_{l+1} \Delta_{+}\left(C_{j}^{n+\eta_{l}}\left|\Delta_{+} U_{j-1}^{n+\eta_{l}}\right|\right) \\
& +\sum_{l=0}^{M-1} \sigma_{l+1} \Delta_{+}\left(D_{j}^{n+\eta_{l}}\left|\Delta_{+} U_{j}^{n+\eta_{l}}\right|\right)
\end{aligned}
$$


Now, consider the case where $j, j+1 \notin \mathcal{C}^{n}$. Using (2.4),

$$
\begin{aligned}
& \left|U_{j+1}^{n+1}-U_{j}^{n+1}\right|=\mid \Delta_{+} U_{j}^{n+\eta_{M-1}} \\
& +\sigma_{M} \Delta_{+}\left(C_{j}^{n+\eta_{M-1}} \Delta_{+} U_{j-1}^{n+\eta_{M-1}}+D_{j}^{n+\eta_{M-1}} \Delta_{+} U_{j}^{n+\eta_{M-1}}\right) \mid \\
& =\mid\left(1+\sigma_{M}\left(C_{j+1}^{n+\eta_{M-1}}-D_{j}^{n+\eta_{M-1}}\right)\right) \Delta_{+} U_{j}^{n+\eta_{M-1}} \\
& -\sigma_{M} C_{j}^{n+\eta_{M-1}} \Delta_{+} U_{j-1}^{n+\eta_{M-1}} \\
& +\sigma_{M} D_{j+1}^{n+\eta_{M-1}} \Delta_{+} U_{j+1}^{n+\eta_{M-1}} \mid \\
& \leq\left(1+\sigma_{M}\left(C_{j+1}^{n+\eta_{M-1}}-D_{j}^{n+\eta_{M-1}}\right)\right)\left|\Delta_{+} U_{j}^{n+\eta_{M-1}}\right| \\
& -\sigma_{M} C_{j}^{n+\eta_{M-1}}\left|\Delta_{+} U_{j-1}^{n+\eta_{M-1}}\right| \\
& +\sigma_{M} D_{j+1}^{n+\eta_{M-1}}\left|\Delta_{+} U_{j+1}^{n+\eta_{M-1}}\right| \\
& =\left|\Delta_{+} U_{j}^{n+\eta_{M-1}}\right|+\sigma_{M} \Delta_{+}\left(C_{j}^{n+\eta_{M-1}}\left|\Delta_{+} U_{j-1}^{n+\eta_{M-1}}\right|\right) \\
& +\sigma_{M} \Delta_{+}\left(D_{j}^{n+\eta_{M-1}}\left|\Delta_{+} U_{j}^{n+\eta_{M-1}}\right|\right) .
\end{aligned}
$$

Applying this argument repeatedly leads to (4.2).

Analysis of the other two cases presents somewhat more difficulty, but still basically follows [11]. Suppose that $j \in \mathcal{C}^{n}$ and $j+1 \notin \mathcal{C}^{n}$. Beginning with (2.5),

$$
\begin{aligned}
U_{j+1}^{n+1}-U_{j}^{n+1}= & U_{j+1}^{n}-U_{j}^{n} \\
& +\sum_{l=0}^{M-1} \sigma_{l+1}\left[\Delta_{+}\left(C_{j}^{n+\eta_{l}} \Delta_{+} U_{j-1}^{n+\eta_{l}}+D_{j}^{n+\eta_{l}} \Delta_{+} U_{j}^{n+\eta_{l}}\right)\right] \\
= & \sum_{l=0}^{M-1} \sigma_{l+1}\left[\left(U_{j+1}^{n}-U_{j}^{n}\right)-\left(U_{j+1}^{n+\eta_{l}}-U_{j}^{n+\eta_{l}}\right)\right] \\
& +\sum_{l=0}^{M-1} \sigma_{l+1}\left[\left(U_{j+1}^{n+\eta_{l}}-U_{j}^{n+\eta_{l}}\right)\right. \\
& \left.\quad+\Delta_{+}\left(C_{j}^{n+\eta_{l}} \Delta_{+} U_{j-1}^{n+\eta_{l}}+D_{j}^{n+\eta_{l}} \Delta_{+} U_{j}^{n+\eta_{l}}\right)\right] .
\end{aligned}
$$

Now applying (2.4), the first sum becomes

$$
\begin{aligned}
& \sum_{l=0}^{M-1} \sigma_{l+1} {\left[U_{j+1}^{n}-U_{j+1}^{n+\eta_{l}}\right] } \\
&=-\sum_{l=0}^{M-1} \sigma_{l+1} \sum_{k=0}^{l-1} \sigma_{k+1}\left(C_{j+1}^{n+\eta_{k}} \Delta_{+} U_{j}^{n+\eta_{k}}+D_{j+1}^{n+\eta_{k}} \Delta_{+} U_{j+1}^{n+\eta_{k}}\right) \\
&=\sum_{l=0}^{M-1} \sigma_{l+1}\left(\eta_{l}-1\right)\left(C_{j+1}^{n+\eta_{l}} \Delta_{+} U_{j}^{n+\eta_{l}}+D_{j+1}^{n+\eta_{l}} \Delta_{+} U_{j+1}^{n+\eta_{l}}\right),
\end{aligned}
$$


where the order of summation has been interchanged as in [11]. This may be substitued into (4.6) to give

$$
\begin{aligned}
U_{j+1}^{n+1}-U_{j}^{n+1}= & \sum_{l=0}^{M-1} \sigma_{l+1}\left[\left(U_{j+1}^{n+\eta_{l}}-U_{j}^{n+\eta_{l}}\right)\right. \\
\left.+\Delta_{+}\left(C_{j}^{n+\eta_{l}} \Delta_{+} U_{j-1}^{n+\eta_{l}}+D_{j}^{n+\eta_{l}} \Delta_{+} U_{j}^{n+\eta_{l}}\right)\right] & \\
& +\sum_{l=0}^{M-1} \sigma_{l+1}\left(\eta_{l}-1\right)\left(C_{j+1}^{n+\eta_{l}} \Delta_{+} U_{j}^{n+\eta_{l}}+D_{j+1}^{n+\eta_{l}} \Delta_{+} U_{j+1}^{n+\eta_{l}}\right) \\
= & \sum_{l=0}^{M-1} \sigma_{l+1}\left[\left(U_{j+1}^{n+\eta_{l}}-U_{j}^{n+\eta_{l}}\right)\right. \\
& \quad+\eta_{l+1} C_{j+1}^{n+\eta_{l}} \Delta_{+} U_{j}^{n+\eta_{l}}+\eta_{l+1} D_{j+1}^{n+\eta_{l}} \Delta_{+} U_{j+1}^{n+\eta_{l}} \\
& \left.\quad-C_{j}^{n+\eta_{l}} \Delta_{+} U_{j-1}^{n+\eta_{l}}-D_{j}^{n+\eta_{l}} \Delta_{+} U_{j}^{n+\eta_{l}}\right] \\
= & \sum_{l=0}^{M-1} \sigma_{l+1}\left[\Delta_{+} U_{j}^{n+\eta_{l}}\left(1+\eta_{l+1} C_{j+1}^{n+\eta_{l}}-D_{j}^{n+\eta_{l}}\right)\right. \\
& \left.\quad+\eta_{l+1} D_{j+1}^{n+\eta_{l}} \Delta_{+} U_{j+1}^{n+\eta_{l}}-C_{j}^{n+\eta_{l}} \Delta_{+} U_{j-1}^{n+\eta_{l}}\right] .
\end{aligned}
$$

Now, using the CFL condition, the following bound can be made:

$$
\begin{aligned}
\left|U_{j+1}^{n+1}-U_{j}^{n+1}\right| \leq \sum_{l=0}^{M-1} \sigma_{l+1} & {\left[\left(1+\eta_{l+1} C_{j+1}^{n+\eta_{l}}-D_{j}^{n+\eta_{l}}\right)\left|\Delta_{+} U_{j}^{n+\eta_{l}}\right|\right.} \\
& \left.+\eta_{l+1} D_{j+1}^{n+\eta_{l}}\left|\Delta_{+} U_{j+1}^{n+\eta_{l}}-C_{j}^{n+\eta_{l}}\right| \Delta_{+} U_{j-1}^{n+\eta_{l}} \mid\right] \\
=\sum_{l=0}^{M-1} \sigma_{l+1}\left[\left|\Delta_{+} U_{j}^{n+\eta_{l}}\right|\right. & \\
+ & \left(\eta_{l+1} C_{j+1}^{n+\eta_{l}}\left|\Delta_{+} U_{j}^{n+\eta_{l}}\right|-C_{j}^{n+\eta_{l}}\left|\Delta_{+} U_{j-1}^{n+\eta_{l}}\right|\right) \\
& \left.+\left(\eta_{l+1} D_{j+1}^{n+\eta_{l}}\left|\Delta_{+} U_{j+1}^{n+\eta_{l}}\right|-D_{j}^{n+\eta_{l}}\left|\Delta_{+} U_{j}^{n+\eta_{l}}\right|\right)\right] .
\end{aligned}
$$

Next consider the term $\left|\Delta_{+} U_{j}^{n+\eta_{l}}\right|$. Using the predictor and the CFL condition,

$$
\begin{aligned}
&\left|\Delta_{+} U_{j}^{n+\eta_{l}}\right|=\mid \Delta_{+} U_{j}^{n+\eta_{l-1}}+\sigma_{l+1} C_{j+1}^{n+\eta_{l-1}} \Delta_{+} U_{j}^{n+\eta_{l-1}} \\
&+\sigma_{l+1} D_{j+1}^{n+\eta_{l-1}} \Delta_{+} U_{j+1}^{n+\eta_{l-1}} \mid \\
& \leq\left(1+\sigma_{l+1} C_{j+1}^{n+\eta_{l-1}}\right)\left|\Delta_{+} U_{j}^{n+\eta_{l-1}}\right| \\
& \quad+\sigma_{l+1} D_{j+1}^{n+\eta_{l-1}}\left|\Delta_{+} U_{j+1}^{n+\eta_{l-1}}\right| \\
&=\left|\Delta_{+} U_{j}^{n+\eta_{l-1}}\right|+\sigma_{l+1} C_{j+1}^{n+\eta_{l-1}}\left|\Delta_{+} U_{j}^{n+\eta_{l-1}}\right| \\
&+\sigma_{l+1} D_{j+1}^{n+\eta_{l-1}}\left|\Delta_{+} U_{j+1}^{n+\eta_{l-1}}\right| .
\end{aligned}
$$

This argument can be applied repeatedly to arrive at

$$
\left|\Delta_{+} U_{j}^{n+\eta_{l}}\right| \leq\left|\Delta_{+} U_{j}^{n}\right|+\sum_{k=0}^{l-1} \sigma_{k+1}\left[C_{j+1}^{n+\eta_{k}}\left|\Delta_{+} U_{j}^{n+\eta_{k}}\right|+D_{j+1}^{n+\eta_{k}}\left|\Delta_{+} U_{j+1}^{n+\eta_{k}}\right|\right] .
$$


So then,

$$
\begin{aligned}
& \sum_{l=0}^{M-1} \sigma_{l+1}\left|\Delta_{+} U_{j}^{n+\eta_{l}}\right| \\
& \quad \leq \sum_{l=0}^{M-1} \sigma_{l+1}\left[\left|\Delta_{+} U_{j}^{n}\right|+\sum_{k=0}^{l-1} \sigma_{k+1}\left[C_{j+1}^{n+\eta_{k}}\left|\Delta_{+} U_{j}^{n+\eta_{k}}\right|+D_{j+1}^{n+\eta_{k}}\left|\Delta_{+} U_{j+1}^{n+\eta_{k}}\right|\right]\right] \\
& \quad=\left|\Delta_{+} U_{j}^{n}\right|+\sum_{l=0}^{M-1} \sigma_{l+1} \sum_{k=0}^{l-1} \sigma_{k+1}\left[C_{j+1}^{n+\eta_{k}}\left|\Delta_{+} U_{j}^{n+\eta_{k}}\right|+D_{j+1}^{n+\eta_{k}}\left|\Delta_{+} U_{j+1}^{n+\eta_{k}}\right|\right] \\
& \quad=\left|\Delta_{+} U_{j}^{n}\right|+\sum_{l=0}^{M-1} \sigma_{l+1}\left(1-\eta_{l+1}\right)\left[C_{j+1}^{n+\eta_{l}}\left|\Delta_{+} U_{j}^{n+\eta_{l}}\right|+D_{j+1}^{n+\eta_{l}}\left|\Delta_{+} U_{j+1}^{n+\eta_{l}}\right|\right]
\end{aligned}
$$

This now may be used in (4.9) to yield (4.2).

The other case, in which $j \notin \mathcal{C}^{n}, j+1 \in \mathcal{C}^{n}$, follows by an analagous argument. With this inequality established in the four possible cases, the TVD result follows immediately by summing on $j$.

Remark. This result holds for the cell averages in the discontinuous Galerkin method, so TVD should be replaced by TVDM in the statement of the proposition as in [2].

\section{AN ENTROPY CONDITION}

Finally, (2.4)-(2.5) satisfy an entropy condition for a smooth convex entropy function. When the advective flux is convex, constructing a scheme which satisfies this condition for a single such entropy function is sufficient to give convergence of the solution to the unique entropy solution. The entropy condition presented here is based on Osher's argument found in [9, 10.

To begin, write the corrector (2.5) as

$$
D_{+}^{t} U_{j}^{n}+\sum_{l=0}^{M-1} \sigma_{l+1} D_{+}^{x} h_{j-\frac{1}{2}}^{n+\eta_{l}}=0,
$$

where $D_{+}^{t} a_{j}^{n}=\frac{a_{j}^{n+1}-a_{j}^{n}}{\Delta t^{n}}$ and $D_{+}^{x} a_{j}^{n}=\frac{a_{j+1}^{n}-a_{j}^{n}}{\Delta x_{j}}$.

Now let $V$ be a convex, twice-differentiable entropy function and $F$ its associated entropy flux. As in [9, 10, an approximate entropy flux is introduced, but this time at each discrete time level:

$$
\Delta_{+} \tilde{F}\left(U_{j}^{n+\eta_{l}}\right) \equiv \Delta_{+}\left(V_{w}\left(U_{j}^{n+\eta_{l}}\right)\left[h_{j}^{n+\eta_{l}}-f\left(U_{j}^{n+\eta_{l}}\right)\right]\right)+\Delta_{+} F\left(U_{j}^{n+\eta_{l}}\right) .
$$

Consider the case $j \in \mathcal{C}^{n}$. Multiply (5.1) by $\Delta x_{j} V_{w}\left(U_{j}^{n}\right)$. Then

$$
\begin{aligned}
\Delta x_{j} V_{w}\left(U_{j}^{n}\right) D_{+}^{t} U_{j}^{n} & =-\left(\sum_{l=0}^{M-1} \sigma_{l+1} D_{+}^{x} h_{j-\frac{1}{2}}^{n+\eta_{l}}\right) V_{w}\left(U_{j}^{n}\right) \Delta x_{j} \\
& =-\left(\sum_{l=0}^{M-1} \sigma_{l+1} V_{w}\left(U_{j}^{n+\eta_{l}}\right) \Delta_{+} h_{j}^{n+\eta_{l}}\right),
\end{aligned}
$$


where the predictor has been used to write $V_{w}\left(U_{j}^{n+\eta_{l}}\right)=V_{w}\left(U_{j}^{h}\right)$. Next, add $\sum_{l=0}^{M-1} \sigma_{l+1} \Delta_{+} \tilde{F}\left(U_{j}^{n+\eta_{l}}\right)$ to both sides of (5.3.3)

$$
\begin{aligned}
\Delta x_{j}\left(V_{w}\left(U_{j}^{n}\right) D_{+}^{t}\right. & \left.U_{j}^{n}+\sum_{l=0}^{M-1} D_{+}^{x} \tilde{F}\left(U_{j}^{n+\eta_{l}}\right)\right) \\
= & \sum_{l=0}^{M-1} \sigma_{l+1} V_{w}\left(U_{j}^{n+\eta_{l}}\right) \Delta_{+} h_{j}^{n+\eta_{l}} \\
& +\sum_{l=0}^{M-1} \sigma_{l+1} \Delta_{+}\left(V_{w}\left(U_{j}^{n+\eta_{l}}\right)\left[h_{j}^{n+\eta_{l}}-f\left(U_{j}^{n+\eta_{l}}\right)\right]\right) \\
& +\sum_{l=0}^{M-1} \sigma_{l+1} \Delta_{+} F\left(U_{j}^{n+\eta_{l}}\right) .
\end{aligned}
$$

The predictor can be used to write the left hand side of (5.4)

$$
\Delta x_{j} \sum_{l=0}^{M-1}\left[\frac{U_{j}^{n+\eta_{l+1}}-U_{j}^{n+\eta_{l}}}{\Delta t^{n}} V_{w}\left(U_{j}^{n+\eta_{l}}\right)+D_{+}^{x} \tilde{F}\left(U_{j}^{n+\eta_{l}}\right)\right] .
$$

Now, using the fundamental theorem of calculus and integration by parts on the right hand side as in 9] leads to

$$
\begin{aligned}
\Delta x_{j} \sum_{l=0}^{M-1}\left[\frac{U_{j}^{n+\eta_{l+1}}-U_{j}^{n+\eta_{l}}}{\Delta t^{n}} V_{w}\left(U_{j}^{n+\eta_{l}}\right)+D_{+}^{x} \tilde{F}\left(U_{j}^{n+\eta_{l}}\right)\right] \\
=\sum_{l=0}^{M-1} \sigma_{l+1} \int_{U_{j}^{n+\eta_{l}}}^{U_{j+1}^{n+\eta_{l}}} \mathrm{~d} w V_{w w}\left[h_{j+\frac{1}{2}}^{n+\eta_{l}}-f\left(U_{j}^{n+\eta_{l}}\right)\right] .
\end{aligned}
$$

Note that the first term on the left hand side is actually $\sigma_{l+1}$ times the divided time difference at $U_{j}^{n+\eta_{l}}$ times $V_{w}\left(U_{j}^{n+\eta_{l}}\right)$. It is not hard to see that this sum of this term over $l$ converges weakly to $\frac{d}{d t} V(u)$.

Now, consider the case $j \notin \mathcal{C}^{n}$. Using the predictor,

$$
U_{j}^{n+1}=U_{j}^{n+\eta_{M-1}}-\sigma_{M-1} \lambda_{j}^{n} \Delta_{+} h_{j-\frac{1}{2}}^{n+\eta_{M-1}}
$$

and multiplying each side by $\Delta x_{j} V_{w}\left(U_{j}^{n+\eta_{M-1}}\right)$ gives

$$
\Delta x_{j} \frac{U_{j}^{n+1}-U_{j}^{n+\eta_{M-1}}}{\Delta t^{n}} V_{w}\left(U_{j}^{n+\eta_{M-1}}\right)=-\sigma_{M-1}\left(\Delta_{+} h_{j}^{n+\eta_{M-1}}\right) .
$$


Add $\sigma_{M-1} \Delta_{+} \tilde{F}\left(U_{j}^{n+\eta_{M-1}}\right)$ to both sides of the equation, rearrange terms, and

$$
\begin{gathered}
\Delta x_{j}\left[\frac{U_{j}^{n+1}-U_{j}^{n+\eta_{M-1}}}{\Delta t^{n}} V_{w}\left(U_{j}^{n+\eta_{M-1}}\right)+D_{+}^{x} \tilde{F}\left(U_{j}^{n+\eta_{M-1}}\right)\right] \\
=\sigma_{M-1}\left[\left(\Delta_{+} V_{w}\left(U_{j}^{n+\eta_{M-1}}\right)\right) h_{j+\frac{1}{2}}^{n+\eta_{M-1}}\right. \\
\left.\quad-\Delta_{+}\left(V_{w}\left(U_{j}^{n+\eta_{M-1}}\right) f\left(U_{j}^{n+\eta_{M-1}}\right)-F\left(U_{j}^{n+\eta_{M-1}}\right)\right)\right] \\
=\sigma_{M-1} \int_{U_{j}^{n+\eta_{l}}}^{U_{j+1}^{n+\eta_{l}}} \mathrm{~d} w V_{w w}\left[h_{j+\frac{1}{2}}^{n+\eta_{l}}-f\left(U_{j}^{n+\eta_{l}}\right)\right] .
\end{gathered}
$$

Finally, summing over $l$ finishes the bound. This inequality has now been established for both $j \in \mathcal{C}^{n}$ and $j \notin \mathcal{C}^{n}$. By multiplying both sides by a nonnegative test function $\phi \in C_{0}^{1}$ and by $\Delta t^{n}$ and summing on $j$ and $n$, it is seen that the entropy condition is satisfied.

Moreover, for convex advective flux it is only necessary to satisfy this condition for a single entropy function $V$ in order to prove convergence of the method to the entropy solution. Several authors, such as [10, have shown how to make appropriate modifications to slope and flux limiters in order to satisfy this condition for the entropy function $V(w)=\frac{w^{2}}{2}$.

\section{Conclusions And Future Work}

Allowing spatially varying time steps in upwind methods helps to resolve the multiscale issues while maintaining essential theoretical properties. Under appropriate local limiting and CFL conditions, these methods satisfy a maximum principle and the TVD property. In addition, additional limiting guarantees convergence to the proper entropy condition for convex flux functions.

Several avenues for continued research on local time stepping remain open. First, the present analysis should be extended to multilevel (more than two) time stepping schemes and to second order in time methods. Research is currently underway to implement this local time stepping methodology to systems of nonlinear conservation laws in two space dimensions.

\section{REFERENCES}

1. M. Berger and J. Oliger, Adaptive mesh refinement for hyperbolic partial differential equations, J. Comp. Phys. 53 (1984), 484-512. MR 85h:65211

2. B. Cockburn and C. Shu, TVB Runge-Kutta local projection discontinuous Galerkin finite element method for scalar conservation laws II: General framework, Math. Comp. 52 (1989), 441-435. MR 90k:65160

3. C. Dawson, High resolution upwind-mixed finite element methods for advection-diffusion equations with variable time-stepping, Numerical Methods for Partial Differential Equations 11 (1995), 525-538. MR 96c:76040

4. C. Dawson and R. Kirby, Higher order schemes for conservation laws with locally varying time steps, SIAM J. Sci. Comput. 22 (2000), 2256-2281. MR 2002f:65115

5. J. E. Flaherty, R. M. Loy, M. S. Shephard, B. K. Szymanski, J. D. Teresco, and L. H. Ziantz, Adaptive local refinement with octree load-balancing for the parallel solution of threedimensional conservation laws, Journal of Parallel and Distributed Computing 47 (1997), $139-152$.

6. A. Harten, High resolution schemes for hyperbolic conservation laws, J. Comp. Phys. 49 (1983), 357-393. MR 84g:65115 
7. R. Kirby, Local time stepping and a posteriori error estimates for flow and transport in porous media, Ph.D. thesis, The University of Texas at Austin, 2000.

8. R. Lowrie, P. Roe, and B. van Leer, Space-time methods for hyperbolic conservation laws, Barriers and challenges in computational fluid dynamics (V. Venkatakrishnan, Manuel D. Salas, and Sukumar R. Chakravarthy, eds.), Kluwer Academic Publishers Group, Dordrecht, 1998, pp. 79-98. CMP 98:09

9. S. Osher, Riemann solvers, the entropy condition, and difference approximations, SIAM J. Numer. Anal. 21 (1984), no. 2, 217-235. MR 86d:65119

10. - Convergence of generalized MUSCL schemes, SIAM J. Numer. Anal. 22 (1985), no. 5, 947-961. MR 87b:65147

11. S. Osher and R. Sanders, Numerical approximations to nonlinear conservation laws with locally varying time and space grids, Math. Comp. 41 (1983), no. 164, 321-336. MR 85i:65121

12. P. K. Sweby, High resolution schemes using flux limiters for hyperbolic conservation laws, SIAM J. Numer. Anal. 21 (1984), 995-1011. MR 85m:65085

13. B. van Leer, Towards the ultimate conservative difference scheme V: A second order sequel to Godunov's method, J. Comp. Phys. 32 (1979), 227-248. CMP 98:05

Department of Computer Science, The University of Chicago, 1100 E. 58th St., Chichago, Illinois 60637

E-mail address: kirby@cs.uchicago.edu 